\title{
Performance Evaluation of a Mixed Vehicular Network with CAM-DCC and LIMERIC Vehicles
}

\author{
Bin Cheng*, Ali Rostami*, Marco Gruteser* John B. Kenney ${ }^{\dagger}$ Gaurav Bansal ${ }^{\dagger}$ and Katrin Sjoberg ${ }^{\ddagger}$ \\ ${ }^{*}$ Winlab, Rutgers University, USA \\ $\dagger$ Toyota Info Technology Center, USA \\ $\ddagger$ Volvo Group Trucks Technology, Sweden \\ Email: $\{$ cb3974, rostami, gruteser\}@winlab.rutgers.edu, \{jkenney, gbansal\}@us.toyota-itc.com, katrin.sjoberg@volvo.com
}

\begin{abstract}
Channel congestion is one of the major challenges for IEEE 802.11p-based vehicular ad hoc networks. Facing the challenge, several algorithms have been proposed. Two good representatives are the Decentralized Congestion Control (DCC) framework defined by ETSI and LIMERIC, a linear control algorithm. Both algorithms control message transmission rate as a function of channel load (i.e., channel busy percentage, CBP). In this work, DCC is assumed to be deployed for day one applications and LIMERIC is introduced into the network afterwards. Given such a mixed vehicular network with vehicles running either DCC or LIMERIC, we evaluate the performance of the two algorithms in the mixed scenario and study the impact of such mixed network operation on the performance through ns-2 simulations. It is observed that converting some CAM-DCC vehicles to LIMERIC vehicles will not lead to any significant performance degradation of either CAM-DCC or LIMERIC vehicles. Any observed performance degradation is small enough so that we would expect typical DSRC applications to still be feasible. Performance differences between DCC and LIMERIC exist but they can be reduced through careful selection of the specific parameters of LIMERIC and DCC, respectively.
\end{abstract}

\section{INTRODUCTION}

Vehicle-to-vehicle communication (V2V) is expected to greatly improve road traffic safety and road traffic efficiency. In recent years, several standards for communication between vehicles have been finalized and V2V communication is moving closer to deployment in different regions of the world. The Medium Access Control (MAC) and Physical Layer (PHY) protocols are specified in the IEEE 802.11p standard [1] and is the wireless technology that has been selected for V2V communication at $5.9 \mathrm{GHz}$ (e.g., Europe and US). However, differences exist in for example higher layer protocols. Early on, it was identified that congestion control was a necessity to avoid unstable network behavior once there is a high number of $\mathrm{V} 2 \mathrm{~V}$ equipped vehicles. This is due to the selected MAC scheme being a carrier sense multiple access with collision avoidance (CSMA/CA) and the ad hoc nature of the network where all vehicles will constantly produce data to be broadcasted several times per second on a common frequency channel. Several congestion control algorithms are under investigation to increase throughput, reduce packet error rate (PER) and decrease inter-packet gap (IPG). It is expected that standards for congestion control algorithms will differ across regions. Two representative algorithms are the Decentralized Congestion Control (CAM-DCC) framework defined in 2011 by the European Telecommunication Standards

978-1-4799-7492-4/15/\$31.00 (C) 2015 IEEE
Institute (ETSI) [2] and the LIMERIC linear control algorithm [3].

With improved algorithms likely to be developed over time, situations could arise where vehicles with different algorithms operate in the same network, referred to as mixed network herein. In particular, there may be a desire to migrate from an earlier deployed congestion control algorithm to a more sophisticated one. One can expect that congestion control algorithms will be refined in the future and that algorithms with better performance will emerge. During a transition period, newly deployed vehicles with the updated algorithm may have to co-exist with vehicles using the legacy algorithm. Other reasons for studying mixed networks could also include the accidental introduction of vehicles with a different algorithm into a region, perhaps through illegal imports or the desire by vehicle manufacturers to minimize differences in their models across regions. In the latter case, a manufacturer may ask permission to deliver vehicles with their standard algorithm to regions initially using a different algorithm.

In this work, the performance impact of such mixed network operation and techniques to reduce undesirable effects is studied using a scenario in which DCC is deployed for day one applications and LIMERIC is introduced afterwards. Given such a mixed network with coexisting DCC and LIMERIC vehicles, we ask the following questions:

- Do the DCC vehicles experience performance changes after LIMERIC vehicles are introduced into the network? Does LIMERIC show a similar performance as DCC?

- If a performance difference does exist, how can it be reduced?

To answer these questions, simulations using ns-2.34 network simulator have been conducted. In these simulations, the percentage of LIMERIC vehicles in the mixed network starts at $0 \%$ (a network with all vehicles running DCC) and increases up to $100 \%$ (a network with all vehicles running LIMERIC) to investigate the performance impact. Simulations are also executed with different LIMERIC target rate and DCC look-up table parameter settings to study the impact of these adjustments on the algorithm performance and their effectiveness in reducing the performance difference between the algorithms.

Following this introduction, Section II explains the CAMDCC and LIMERIC algorithms. The configuration of simula- 
tions is described in Section III. The simulation results are presented in Section IV, and the discussion of the results is presented in Section V. We present the conclusions and direction for future work in Section VI.

\section{BACKGROUND}

Most vehicular network applications require frequent exchange of status messages among vehicles, which inform neighbors about vehicle positions and movements. These messages are referred to as Cooperative Awareness Messages (CAMs) in Europe [4] and Basic Safety Messages (BSM) in the US [5]. Such messages are transmitted multiple times per second and when many vehicles enter the same area, the total volume of messages can lead to channel congestion. The congestion control algorithms thus aim to reduce the channel load locally when vehicle density becomes too high, and to avoid unstable network behavior with many concurrent transmissions leading to many packet losses. The algorithms specify when messages can be generated and when they can be sent to the MAC layer for transmission. The CBP is defined as the fraction of time during which the channel is measured as busy and it can be used to indicate the channel condition.

\section{A. CAM-DCC algorithm}

When using DCC for controlling the congestion, it does not only shape the traffic into the MAC layer, but also limits CAM generation in the facilities layer. Therefore, the DCC algorithm with CAM generation is referred to as CAM-DCC in this paper. The CAM generation has been implemented in the simulator according to EN 302 637-2 [4]. The specific generation times of CAMs are determined by vehicle dynamics and can be restricted by DCC if necessary. The time interval between two generated CAMs should be no less than 100 $\mathrm{ms}$ and no more than $1000 \mathrm{~ms}$ (i.e., generation frequency is between 1-10 Hz). Generally, a new CAM shall be generated when the following two conditions are met:

- The elapsed time since the last CAM generation is larger than the message interval, which is provided by DCC through the parameter T_GenCam_DCC.

- One of the following vehicle dynamics criteria is met: 1) heading changed $>4^{\circ}$;2) position changed $>4$ meters; 3) magnitude of speed changed $>0.5 \mathrm{~m} / \mathrm{sec}$

If the above two conditions are not met for 1 second after the last CAM generation, a CAM is generated immediately. When a CAM is generated due to one of these dynamics criteria, a second and third (the actual number is controlled by $N \_G e n C a m$. The maximum value of $N \_G e n C a m$ defined in $\overline{E N} 302637-2$ is 3), CAM shall be generated at the same interval unless the changing dynamics lead to a shorter interval.

DCC regulates CAM generation by using a state machine, which distinguishes between three main states: RELAXED, ACTIVE, and RESTRICTIVE. The ACTIVE state can be further divided into several sub-states. Each state (sub-state) defines a set of transmission parameters (e.g. transmit rate, transmit power, data rate), which controls the transmission behaviors. Herein, only transmit rate is considered for controlling the channel load. The transition between different states are driven by the channel load, locally measured by each vehicle during a sampling interval. To avoid the DCC algorithm from jumping between states after every CBP period, an inertia is introduced to the system in the form of two parameters (i.e., NDL_TimeUp and NDL_TimeDown) dictating for how long the DCC algorithm shall at least stay in one state.

\section{B. LIMERIC algorithm}

LIMERIC is a distributed and adaptive linear rate-control algorithm where each vehicle adapts its message rate in a way such that the total channel load converges to a specified target [3]. The message rate of vehicle $j$ (denoted as $r_{j}(t)$ ) is adapted every $\theta$ time using the following linear equation:

$$
r_{j}(t)=(1-\alpha) r_{j}(t-\theta)+\beta\left(C B P_{T}-C B P_{m}(t-\theta)\right)
$$

Where $C B P_{T}$ denotes the target channel load. $\alpha$ and $\beta$ are adaption parameters that control stability, fairness and steady state convergence. For a $C B P_{T}$, in steady state, LIMERIC converges to a CBP determined by:

$$
C B P_{\text {con }}=\frac{K \beta}{20 \alpha+K \beta} \times C B P_{T}
$$

Where $K$ is the number of vehicles within the interference range. According to the equation, the converged channel load can be adjusted by using different $C B P_{T}$. In LIMERIC, a message will be generated when there are channel resources available at least with $1 \mathrm{~Hz}$, which is the maximum time between two generations (same as in the CAM case).

\section{Simulation SET-UP}

In this section, the DCC and LIMERIC algorithms are investigated through simulations. The SUMO mobility simulator has been configured for a highway of length $4 \mathrm{~km}$, with 3 lanes in each direction. As shown in Figure 1, the middle part of the road is a winding section of linear length $375 \mathrm{~m}$ (with a radius of the winding part set to be $40 \mathrm{~m}$ ). When vehicles are traveling on this winding section, the number of vehicles within the neighborhood becomes larger than when the vehicles are moving on the straight highway. The increased number of neighbors can then lead to a growth in the channel load. Hence, the road topology allows us to study the performance of the congestion control algorithms when the vehicles are experiencing changed channel load.

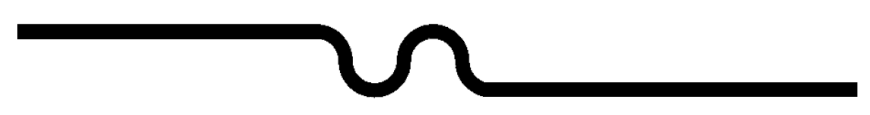

Fig. 1: Road topology for simulations

The average speeds of vehicles on the 3 lanes are $19 \mathrm{~m} / \mathrm{s}$ in the fast (inside) lane, $18 \mathrm{~m} / \mathrm{s}$ in the middle lane, and 17 $\mathrm{m} / \mathrm{s}$ in the slowest (outside) lane. When a vehicle reaches the end of the road, it will loop around and then move in the opposite direction. The number of vehicles in the simulation is set to 1000 , and the start position of each vehicle is uniformly distributed on the road. Each vehicle randomly chooses a time for the first transmission in the range between $0 \mathrm{sec}$ to $0.5 \mathrm{sec}$ after the simulation starts.

A typical DSRC transmission range in highway scenarios is $500 \mathrm{~m}$. To create such range in simulations with the default 
ns-2 Nakagami propagation model, we set the transmission power to $10 \mathrm{dBm}$ and use the same fading parameters as in [6]. Although more precise propagation model calibrations are possible, we believe that this model is sufficient to evaluate the congestion control algorithm interactions. In reality, a higher transmit power may be needed for the same transmission range. The CBP is measured periodically by each vehicle and the measurement period is synchronized across all vehicles in the simulations both for CAM-DCC and LIMERIC. The length of the CBP measurement period is $100 \mathrm{~ms}$. The configuration for all simulations are listed in Table I.

TABLE I: Simulation parameters

\begin{tabular}{|c|c|}
\hline Parameter & Value \\
\hline Noise floor & $-99 \mathrm{dBm}$ \\
\hline Carrier sense threshold & $-96 \mathrm{dBm}$ \\
\hline $\begin{array}{c}\text { Packet Reception SINR } \\
\text { (for 6Mbps datarate) }\end{array}$ & $7 \mathrm{dBm}$ \\
\hline$C W_{\text {min }}$ & 15 \\
\hline AIFSN & 2 \\
\hline Facilities layer payload & $350 \mathrm{byte}$ \\
\hline Transmission Rate & $6 \mathrm{Mbps}$ \\
\hline Transmission Power & $10 \mathrm{dBm}$ \\
\hline GPS Update Frequency & $10 \mathrm{~Hz}$ \\
\hline $\begin{array}{c}\text { CBP measurement period } \\
\left(T \_C B P \_u p d a t e\right)\end{array}$ & $100 \mathrm{msec}$ \\
\hline Simulation time & $200 \mathrm{sec}$ \\
\hline Number of Vehicles & 1000 \\
\hline
\end{tabular}

\section{A. DCC configuration}

Two different parameter settings have been used for CAMDCC in the simulations to evaluate the performance of the mixed scenario. Recall that CAM-DCC relies on a table look-up and state machine to map channel load measures to transmission rates. Table II shows setting 1 and Table III shows setting 2 . The difference between the two settings is that setting 2 allows for slightly higher transmit rates given a specific channel load.

TABLE II: CAM-DCC look-up table, setting 1

\begin{tabular}{|l|c|l|l|}
\hline $\begin{array}{l}\text { Channel } \\
\text { Load }\end{array}$ & State & $\begin{array}{l}\text { Packet } \\
\text { Interval }\end{array}$ & $\begin{array}{l}\text { Packet Tx } \\
\text { Rate }\end{array}$ \\
\hline$<\mathbf{3 0 \%}$ & RELAXED & $100 \mathrm{~ms}$ & $10 \mathrm{~Hz}$ \\
\hline $\mathbf{3 0 - 3 9 \%}$ & Active 1 & $200 \mathrm{~ms}$ & $5 \mathrm{~Hz}$ \\
\hline $\mathbf{4 0 - 4 9 \%}$ & Active 2 & $300 \mathrm{~ms}$ & $3.33 \mathrm{~Hz}$ \\
\hline $\mathbf{5 0 - 5 9 \%}$ & Active 3 & $400 \mathrm{~ms}$ & $2.5 \mathrm{~Hz}$ \\
\hline$\geq \mathbf{6 0}$ & RESTRICTED & $500 \mathrm{~ms}$ & $2 \mathrm{~Hz}$ \\
\hline
\end{tabular}

TABLE III: CAM-DCC look-up table, setting 2

\begin{tabular}{|l|c|l|l|}
\hline $\begin{array}{l}\text { Channel } \\
\text { Load }\end{array}$ & State & $\begin{array}{l}\text { Packet } \\
\text { Interval }\end{array}$ & $\begin{array}{l}\text { Packet Tx } \\
\text { Rate }\end{array}$ \\
\hline$<\mathbf{4 0 \%}$ & RELAXED & $100 \mathrm{~ms}$ & $10 \mathrm{~Hz}$ \\
\hline $\mathbf{4 0 - 4 9 \%}$ & Active 1 & $200 \mathrm{~ms}$ & $5 \mathrm{~Hz}$ \\
\hline $\mathbf{5 0 - 5 9 \%}$ & Active 2 & $300 \mathrm{~ms}$ & $3.33 \mathrm{~Hz}$ \\
\hline $\mathbf{6 0 - 6 9 \%}$ & Active 3 & $400 \mathrm{~ms}$ & $2.5 \mathrm{~Hz}$ \\
\hline$\geq \mathbf{7 0}$ & RESTRICTED & $500 \mathrm{~ms}$ & $2 \mathrm{~Hz}$ \\
\hline
\end{tabular}

\section{B. LIMERIC configuration}

In the LIMERIC implementation, vehicles generate CAMs when LIMERIC allows (i.e., the CAM generation rules based on vehicle dynamics are not applied). LIMERIC adapts the transmit rate in such a way that the channel load is driven towards the target CBP $\left(C B P_{T}\right)$ and it converges to a CBP value that is slightly lower than the targeted one. Different target CBP values lead to different convergence behaviors for LIMERIC. For studying the impact of the LIMERIC target CBP on the performance of the mixed network, two different settings have been used: in setting 1 , the target, $C B P_{T}$, is set to $79 \%$ and in setting $2 C B P_{T}=68 \%$. The other parameters needed for LIMERIC are set as follows for both CBP targets: $\alpha=0.1, \beta=0.033$, and $\theta=200 \mathrm{~ms}$.

\section{Simulation Results}

The Packet Error Rate (PER) and the 95th percentile InterPacket Gap (IPG) are used as performance evaluation metrics. PER is defined as the number of missed packets at a receiver from a particular transmitter divided by the total number of packets sent by that transmitter. IPG is defined as the elapsed time between consecutive successful packet receptions from a particular transmitter. The 95th percentile is the near worst case of the collected IPGs.

Note that all results provided here are based on transmissions carried out on the winding part of the road and averaged over distance bins according to the distance between the transmitter and the receiver. That is, for a pair of transmitter and receiver, if the transmitter is currently on the winding part of the road when the receiver is receiving packets from that transmitter, the distance between them will be calculated and binned by certain distance (The bin size is $50 \mathrm{~m}$ in all calculations). Each bin collects data from all these pairs and then calculates the metrics. The calculation of the metrics is divided between CAM-DCC transmitters and LIMERIC transmitters. The CAM-DCC results are labeled CAMDCC_x\% and the LIMERIC results are labeled LIMERIC_y\%, where, $\mathrm{x}$ and $y$ indicate the percentage of vehicles running CAM-DCC and that of vehicles running LIMERIC in the mixed network, respectively. If $x+y=100$, the results for CAM-DCC and LIMERIC are obtained from the same mixed network.

\section{A. Packer Error Rate}

Figure 2 (a)-(d), shows the PER for CAM-DCC vehicles and LIMERIC vehicles in both homogeneous and mixed network scenarios. For CAM-DCC, Figures 2(a) and 2(b) use the Table II rates (Setting 1) while 2(c) and 2(d) use the Table III rates (Setting 2). For LIMERIC, Figures 2(a) and 2(c) use CBP target 79\% (Setting 1) while 2(b) and 2(d) use CBP target $68 \%$ (Setting 2). Thus, all four combinations of settings are represented. The PER curves for LIMERIC and CAM-DCC are very close to each other, which indicates good co-existence in the mixed network case. The PER curve for the (homogeneous) $100 \%$ CAM-DCC scenario is a bit higher than the others in part 2(a) and 2(b). This is most likely due to a phenomenon that has been reported in [7], in which DCC state changes lead to synchronized transmissions among neighboring vehicles, resulting in a higher than expected packet collision rate. In the figure we see that this can increase PER by up to approximately 10 percentage points. The elevated PER is not present in the pure LIMERIC or mixed CAM-DCC/LIMERIC networks, nor when CAM-DCC uses rate Table III. This synchronization effect is not the focus of the present paper, and is not discussed in more detail here. 


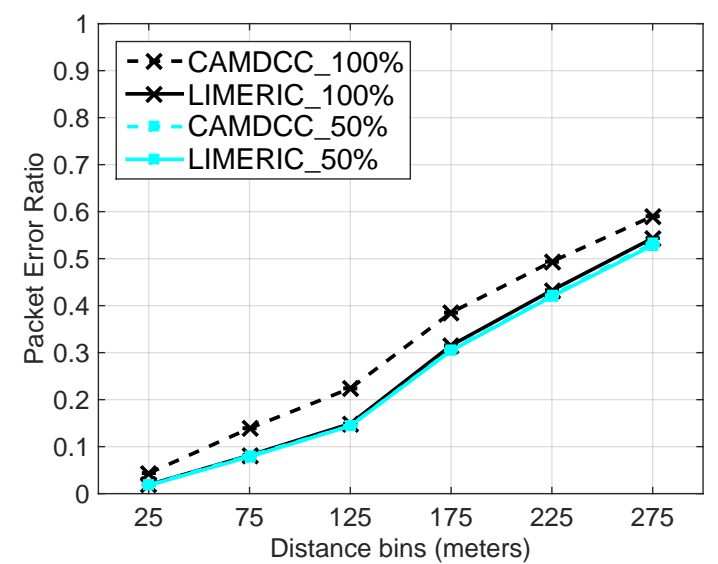

(a)

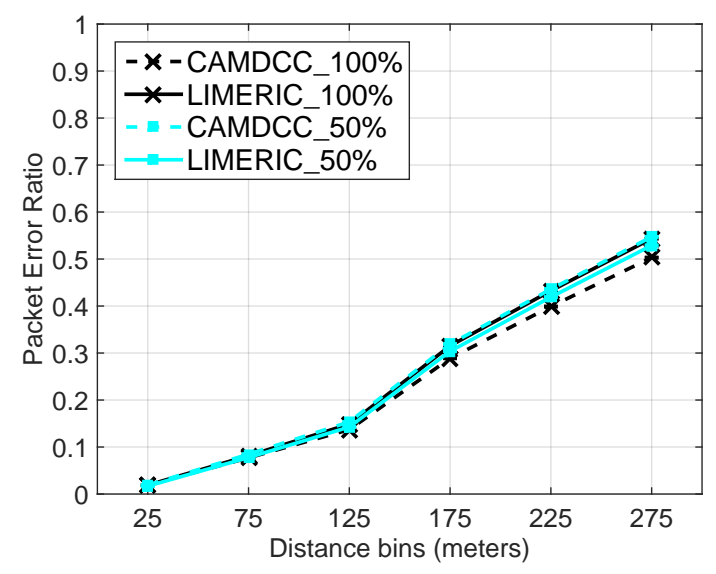

(c)

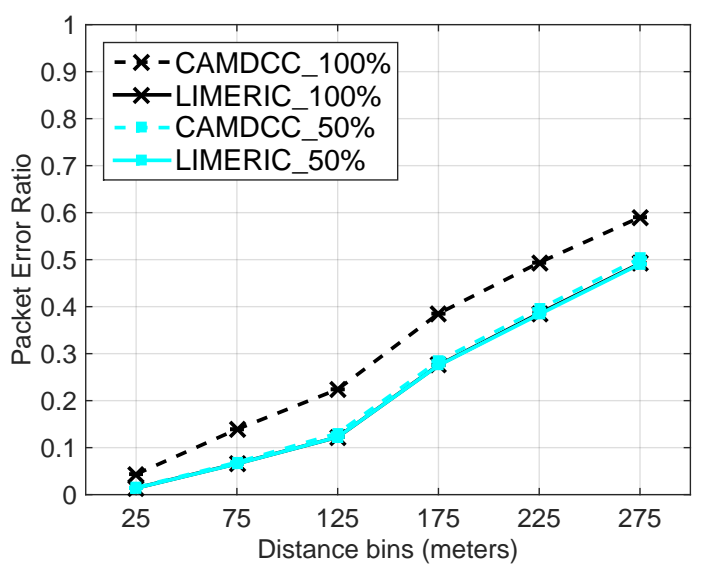

(b)

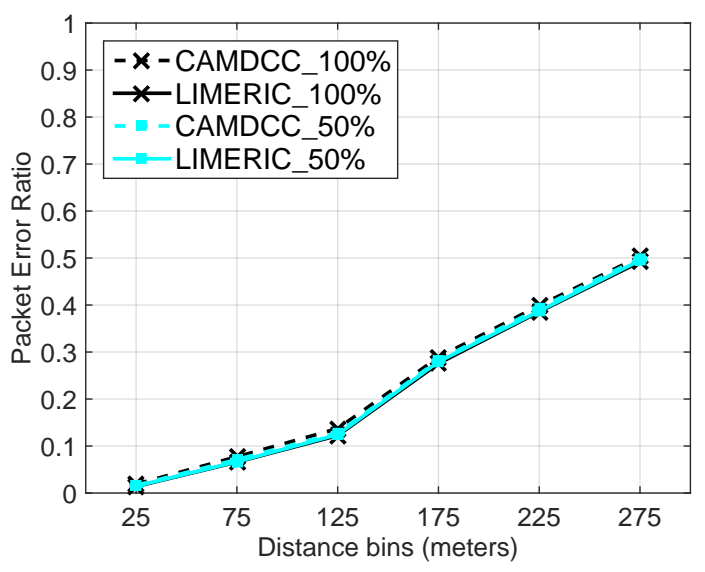

(d)

Fig. 2: PER of the mixed network: (a) CAM-DCC setting 1, LIMERIC setting 1 (CBP $=79 \%$ ); (b) CAM-DCC setting 1, LIMERIC setting $2(\mathrm{CBP}=68 \%)$; (c) CAM-DCC setting 2, LIMERIC setting 1 (CBP $=79 \%)$; (d) CAM-DCC setting 2, LIMERIC setting $2(\mathrm{CBP}=68 \%)$

The vehicles using DCC are subject to CAM generation based on kinetic rules as discussed earlier. Given that the speed of vehicles averages between 17 and $19 \mathrm{~m} / \mathrm{s}$, the generation rules would create between four and five CAMs/second. However, for the vehicle density in the test, the DCC function limits CAM generation below that rate.

The PER is generally lower when LIMERIC is set to a target CBP of $68 \%$. This can be seen by comparing the mixed network and pure LIMERIC results in Figure 2(b) with those in 2(a), and also by comparing the results in 2(d) with those in 2(c). The lower CBP target causes LIMERIC vehicles to converge to a lower rate, which reduces contention for channel access and leads to lower PER.

\section{B. Inter-Packet Gap}

The performance metric IPG shows differences between the different parameter settings of the two studied algorithms. In Figure 3(a)-(b), the IPGs for the networks with different mixed percentages are depicted. In all these simulations, both the CAM-DCC and LIMERIC use setting 1 (i.e., the DCC look-up table is Table II, and the LIMERIC target CBP is
79\%). The IPG for the CAM-DCC vehicles is never lower than 0.5 seconds at the shortest distance bin (and hardly no packet errors exist at this short distance) implying that all these CAM-DCC vehicles in the winding part are in RESTRICTIVE state (CBP is above 60\%) throughout the simulation (giving a transmit rate of $2 \mathrm{~Hz}$ ). When there are only CAM-DCC vehicles (CAMDCC_100\%) in the network, the IPG is more or less increasing linearly but when as little as $20 \%$ of the vehicles are running LIMERIC and the rest is running CAM-DCC $(80 \%)$, there is suddenly a performance gain for the CAMDCC vehicles starting at 75 meters. This can be explained by LIMERIC reducing the number of synchronized transmissions as explained earlier. In the mixed scenarios, when there are both LIMERIC and CAM-DCC vehicles, all penetration rates of CAM-DCC vehicles obtain the same performance. It is because all CAM-DCC vehicles moving on the winding road in these scenarios constantly operate in RESTRICTIVE state. When LIMERIC vehicles are introduced, they try to reach the target CBP of 79\% through more frequent transmissions. That is why the IPG is smaller when there are few LIMERIC vehicles (i.e., LIMERIC_20\%) compared to when the number of LIMERIC vehicles increases (i.e., LIMERIC_80\%), because 


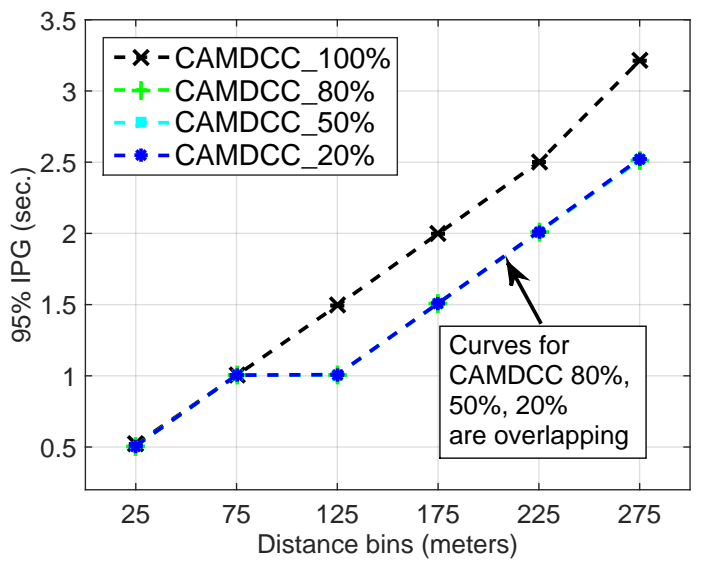

(a)

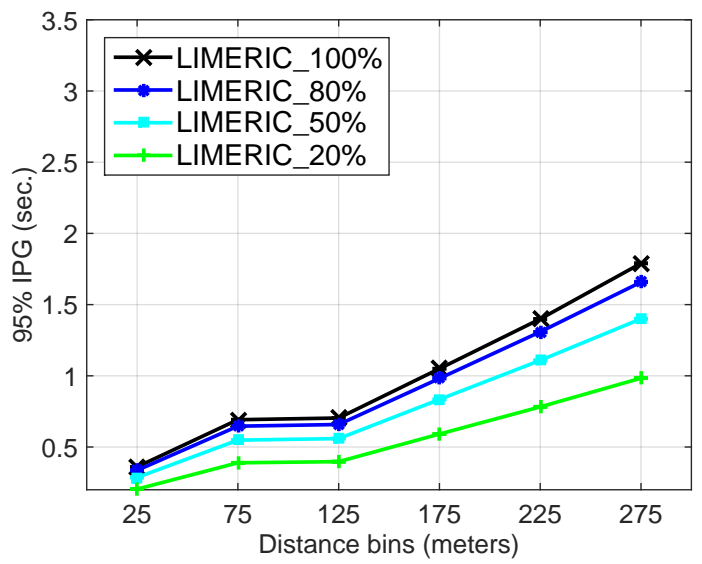

(b)

Fig. 3: 95th percentile IPG of the mixed network with Setting 1 of: (a) CAM-DCC; (b) LIMERIC, respectively.

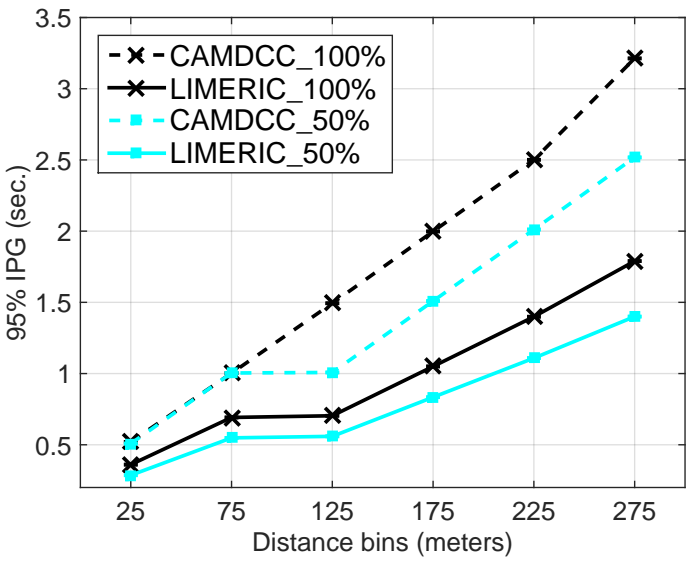

(a)

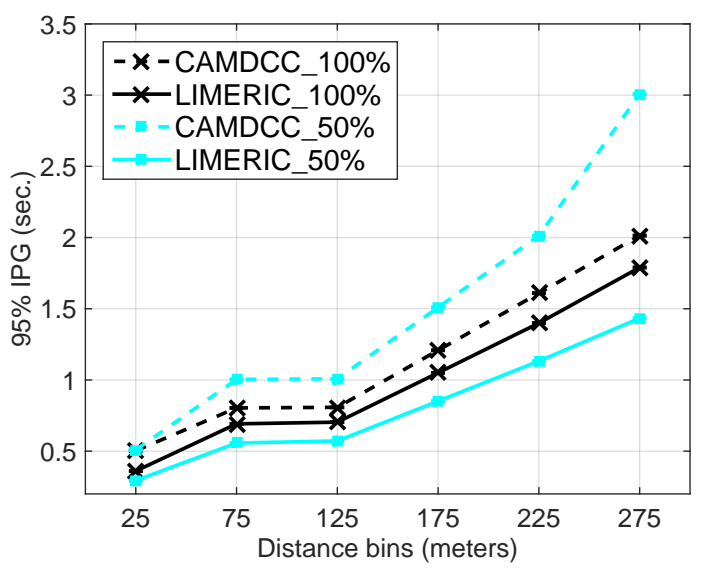

(c)

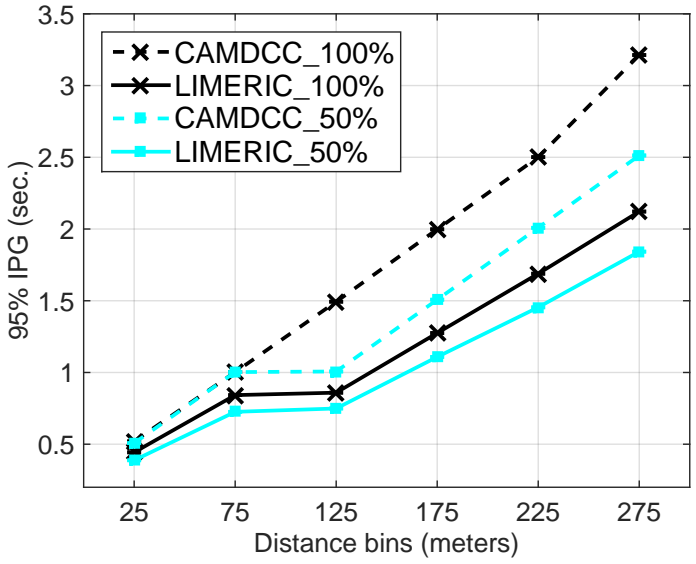

(b)

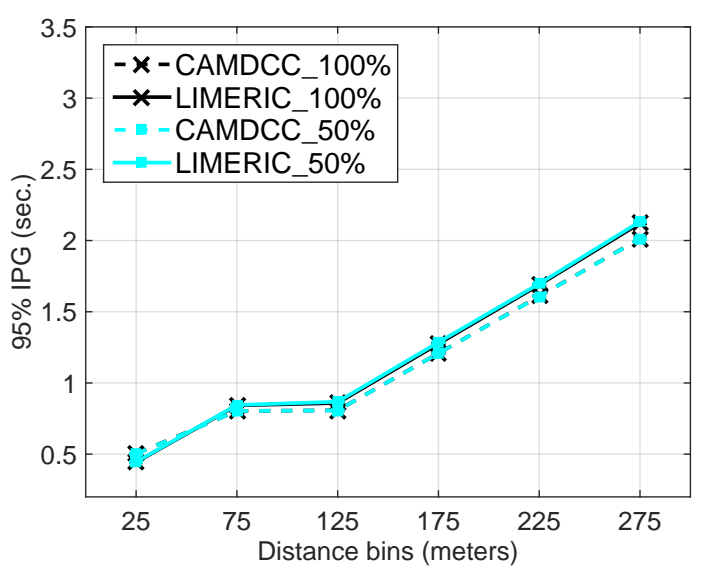

(d)

Fig. 4: 95th percentile IPG of the mixed network: (a) CAM-DCC setting 1, LIMERIC setting 1 (CBP = 79\%); (b) CAM-DCC setting 1, LIMERIC setting $2(\mathrm{CBP}=68 \%)$; (c) CAM-DCC setting 2, LIMERIC setting 1 (CBP = 79\%); (d) CAM-DCC setting 2, LIMERIC setting $2(\mathrm{CBP}=68 \%)$ 
then more LIMERIC vehicles are struggling for the target.

In Figure 4(b), the IPG for CAM-DCC setting 1 (Table II) and LIMERIC setting 2 (target $C B P_{T}=68 \%$ ) is depicted. Here, it is seen that LIMERIC is closing the gap and moves towards the performance of the CAM-DCC algorithm and that is because LIMERIC is not given the same room to fill the channel with packets to reach the target. When aiming at a lower target CBP, each vehicle choose a comparatively lower rate and a lower rate can lead to a larger IPG. However, there is still a performance difference in terms of IPG between CAMDCC and LIMERIC in the mixed scenario explained by the coarse grained mapping of CBP values to transmit rates in the CAM-DCC case.

Setting 2 of the CAM-DCC (Table III) together with LIMERIC setting 1 (target $C B P_{T}=79 \%$ ) is shown in Figure 4(c). Recall that setting 2 of the CAM-DCC pushes vehicles into RESTRICTIVE state above a channel load of 70\%. The channel load for $100 \%$ penetration of CAM-DCC vehicles on the winding part of the road is between 61-69\%, implying that here the vehicles have a transmit rate of $2.5 \mathrm{~Hz}$ (i.e., they are in state Active 3). This decreases the distance between packet transmissions at sender side to $400 \mathrm{msec}$ and CAM-DCC with $100 \%$ penetration are coming closer to the LIMERIC vehicles. However, when there is a fifty-fifty network of CAM-DCC and LIMERIC vehicles, once again, the CAM-DCC vehicles are pushed into RESTRICTIVE state since LIMERIC strives for the CBP target of $79 \%$.

LIMERIC setting $2\left(C B P_{T}=68 \%\right)$ together with CAMDCC setting 2 is shown in Figure 4(d). Here, there is no difference between different algorithm penetrations. LIMERIC is striving towards a CBP target that corresponds to state Active 3 in Table III (transmit rate of $2.5 \mathrm{~Hz}$ ).

\section{Discussion}

The different parameter settings of the two algorithms reveal interesting facts. Perhaps the most surprising finding, is that CAM-DCC performance can be improved by inserting LIMERIC vehicles into the network. This is because CAMDCC suffers from the synchronized CBP measurements. In CAM-DCC, synchronized CBP measurements can lead to many neighboring nodes deciding on a new rate and immediately transmitting at the same time. Moreover, since a range of $\mathrm{CBP}$ values maps to one rate, many neighboring nodes choose the same rate and synchronized transmissions recur over a series of transmissions and last for a long time. The differences in performance for certain settings might disappear if the vehicles had different starting times for their CBP measurement interval. However, we are not aware of the standard defining whether CBP measurements should be synchronized and given the availability of GPS clocks, it is plausibile that implementers would make the synchronized choice.

Note that a key difference between LIMERIC and CAMDCC is how they control the channel load. The former is striving towards a target and controls the target by increasing or decreasing the message rate along a continuum. CAMDCC, on the other hand, is an open-loop with a few discrete rate choices. We believe that this makes CAM-DCC more susceptible to such synchronization effects.

\section{CONCLUSION AND FUture WORK}

This work has examined the coexistence of vehicles running different congestion control algorithms (CAM-DCC and LIMERIC) through simulations of a highway scenario. Congestion control aims to prevent overloading the channel with position announcement messages (termed CAM in Europe and BSM in the US), which are the fundamental messaging primitive for many road traffic safety applications.

The results of the studied scenario suggest that introducing LIMERIC vehicles in a CAM-DCC network does not lead to any major performance degradation for either of the studied algorithms. To the contrary, the performance of CAM-DCC can even be improved through LIMERIC vehicles, because CAM-DCC is affected by synchronization inefficiencies that lead to increased packet collisions in the simulation scenarios. Packet error rate degradations are typically less than $5 \%$ but CAM-DCC PER improvements can be as large as $10 \%$.

Further, the magnitude of the performance differences between LIMERIC and CAM-DCC can be controlled through adjustments in the LIMERIC target CBP and the CAMDCC look-up table parameters. The results show that such adjustments can close the gap in terms of both PER and IPG.

This first study of mixed network operation did not reveal any major performance degradation and shows promise for coexistence of vehicular congestion control algorithms. While the current results are limited to the studied highway scenario, we believe that this conclusion will apply to other scenarios as well. Future work should confirm this.

\section{ACKNOWLEDGEMENT}

This material is based in part upon work at Rutgers University that is supported by Toyota ITC.

\section{REFERENCES}

[1] "IEEE Standard for Information Technology Telecommunications and information exchange between systems Local and metropolitan area networks Specific requirements; Part 11: Wireless LAN Medium Access Control (MAC) and Physical Layer (PHY) specifications; Amendment 6: Wireless Access in Vehicular Environments," July 2010.

[2] ETSI TS 102687 V1.1.1, "Intelligent transport systems (ITS): Decentralized congestion control mechanisms for intelligent transport systems operating in the $5 \mathrm{ghz}$ range; access layer part," July 2011.

[3] Gaurav Bansal, John B. Kenney, and Charles E. Rohrs, "LIMERIC: A linear adaptive message rate algorithm for dsrc congestion control," Vehicular Technology, IEEE Transactions on, vol. 62, no. 9, pp. 41824197, Nov 2013.

[4] ETSI EN 302 637-2 V1.3.2, "Intelligent transport systems (ITS); vehicular communications; basic set of applications; part 2: Specification of cooperative awareness basic service," November 2014.

[5] SAE Std J2735, "Dedicated short range communications (DSRC) message set dictionary," November 2009.

[6] Gaurav Bansal, Hongsheng Lu, John B. Kenney, and Christian Poellabauer, "EMBARC: Error model based adaptive rate control for vehicle-to-vehicle communications," in Proceeding of the Tenth ACM International Workshop on Vehicular Inter-networking, Systems, and Applications. 2013, VANET '13, pp. 41-50, ACM.

[7] Gaurav Bansal, Bin Cheng, Ali. Rostami, Katrin Sjoberg, John B. Kenney, and Marco Gruteser, "Comparing LIMERIC and DCC approaches for VANET channel congestion control," in Wireless Vehicular Communications (WiVeC), 2014 IEEE 6th International Symposium on, Sept 2014, pp. 1-7. 